Article

\title{
On a generalization of KU-algebras pseudo-KU algebras
}

\section{Daniel A. Romano}

International Mathematical Virtual Institute 6, Kordunaška Street, 78000 Banja Luka, Bosnia and Herzegovina.; bato49@hotmail.com

Received: 10 January 2020; Accepted: 1 June 2020; Published: 5 July 2020.

\begin{abstract}
As a generalization of KU-algebras, the notion of pseudo-KU algebras is introduced in 2020 by the author (D. A. Romano. Pseudo-UP algebras, An introduction. Bull. Int. Math. Virtual Inst., 10(2)(2020), 349-355). Some characterizations of pseudo-KU algebras are established in that article. In addition, it is shown that each pseudo-KU algebra is a pseudo-UP algebra. In this paper it is a concept developed of pseudo-KU algebras in more detail and it has identified some of the main features of this type of universal algebras such as the notions of pseudo-subalgebras, pseudo-ideals, pseudo-filters and pseudo homomorphisms. Also, it has been shown that every pseudo-KU algebra is a pseudo-BE algebra. In addition, a congruence was constructed on a pseudo-KU algebra generated by a pseudo-ideal and shown that the corresponding factor-structure is and pseudo-KU algebra as well.
\end{abstract}

Keywords: KU-algebra, Pseudo-KU algebra, pseudo-UP algebra, pseudo-BE algebra, pseudo-ideals, pseudo-homomorphism.

MSC: $62 \mathrm{D} 05$.

\section{Introduction}

$\mathbf{T}$ he concept of pseudo-BCK algebras was introduce in [1] by Georgescu and Iorgulescu as an extension of BCK-algebras. The notion of pseudo-BCI algebras was introduced and analyzed in [2] by Dudek and Jun as a generalization of BCI-algebras. The concept of pseudo-BE algebras was introduced in 2013 and their properties were explored by Borzooei et al., in [3]. These algebraic structures has been in the focus of many authors (for example, see [4-10]). Pseudo BL-algebras are a non-commutative generalization of BL-algebras introduced in [11]. Pseudo BL-algebras are intensively studied by many authors (for example, [12-14]).

Prabpayak and Leerawat 2009 in $[15,16]$ introduced a new algebraic structure which is called KU-algebras. They studied ideals and congruences in KU-algebras. They also introduced the concept of homomorphism of KU-algebras and investigated some related properties. Moreover, they derived some straightforward consequences of the relations between quotient KU-algebras and isomorphism. Many authors took part in the study of this algebraic structure (for example: $[17,18]$ ).

A detailed listing of the researchers and their contributions to these activities it can be found in [19]. Here, we will highlight the contribution of [20]. In [21], Kim and Kim introduced the concept of BE-algebras as a generalization of dual BCK-algebras. This class of algebra was also studied by Rezaei and Saeid 2012 in article [22]. In the article [20], the authors (Rezaei, Saeod and Borzooei) proved that a KU-algebra is equivalent to a commutative self-distributive BE-algebra. (A BE-algebra $A$ is a self-distributive if $x \cdot(y \cdot z)=(z \cdot y) \cdot(x \cdot z)$ for all $x, y, z \in A$.) Additionally, they proved that every KU-algebra is a BE-algebra ([20], Theorem 3.4), every Hilbert algebra is a KU-algebra ([20], Theorem 3.5) and a self-distributive KU-algebra is equivalent to a Hilbert algebra ([20]). Iampan constructed PU-algebra as a generalization of KU-algebra in [19] in 2017 and showed that each KU-algebra is a PU-algebra.

In article [23], the author designed the concepts of pseudo-UP ([23], Definition 3.1) and pseudo-KU-algebras ([23], Definition 4.1) and showed that each pseudo-KU algebra is a pseudo-UP algebra ([23], Theorem 4.1). However, the term 'pseudo KU-algebra' and mark 'PKU' has already been used in [24] for different purposes. It should be noted here that this term 2019 has been renamed to 'JU-algebra' ([25]). Although introducing the term 'pseudo-KU algebra' as a name for a structure constructed in the manner described here and using the abbreviation 'pKU' for this algebra can lead to confusion, we did it for needs of article [23] and of this paper. 
In this paper we develop the concept in more detail of pseudo-KU algebras and we identify some of the main features of this type of universal algebras. The paper was designed as follows: After the Section 2, which outlines the necessary previous terms, Section 3 introduces the concept of pseudo-KU algebra and analyzes some of its important properties. In Section 4, the concept of pseudo-KU algebras is linked to the concepts of pseudo-UP and pseudo-BE algebras. Section 5 deals with some substructures of this class of algebras such as pseudo-subalgebras, pseudo-ideals and pseudo-filters. Finally, in Section 6, the concepts of pseudo-homomorphisms and congruences on pseudo-KU algebras are analyzed.

\section{Preliminaries}

In this section we will describe some elements of KU-algebras from the literature $[15,16]$ necessary for our intentions in this text.

Definition 1. ([15]) An algebra $A=(A, \cdot, 0)$ of type $(2,0)$ is called a KU-algebra where $A$ is a nonempty set, ${ }^{\prime} \cdot{ }^{\prime}$ is a binary operation on $A$, and 0 is a fixed element of $A$ (i.e. a nullary operation) if it satisfies the following axioms:

(KU-1) $(\forall x, y, z \in A)((x \cdot y) \cdot((y \cdot z) \cdot(x \cdot z))=0)$,

$(\mathrm{KU}-2)(\forall x \in A)(0 \cdot x=x)$,

$(\mathrm{KU}-3)(\forall x \in A)(x \cdot 0=0)$, and

(KU-4) $(\forall x, y \in A)((x \cdot y=0 \wedge y \cdot x=0) \Longrightarrow x=y)$.

On a KU-algebra $A=(A, \cdot, 0)$, we define the KU-ordering $\leqslant$ on $A$ as follows ([15], pp. 56):

$$
(\forall x, y \in A)(x \leqslant y \Longleftrightarrow y \cdot x=0) .
$$

Lemma 1. In a KU-algebra $A$, the following properties hold:

(1) $(\forall x \in A)(x \leqslant x)$,

(2) $(\forall x, y \in A)((x \leqslant y \wedge y \leqslant x), \Longrightarrow x=y)$,

(3) $(\forall x, y, z \in A)((x \leqslant y \wedge y \leqslant z) \Longrightarrow x \leqslant z)$,

(4) $(\forall x, y, z \in A)(x \leqslant y \Longrightarrow z \cdot x \leqslant z \cdot y)$,

(5) $(\forall x, y, z \in A)(x \leqslant y \Longrightarrow y \cdot z \leqslant x \cdot z)$,

(6) $(\forall x, y \in A)(x \cdot y \leqslant y)$ and

(7) $(\forall x \in A)(0 \leqslant x)$.

Definition 2. ([15]) Let $S$ be a non-empty subset of a KU-algebra $A$.

(a) The subset $S$ is said to be a KU-subalgebra of $A$ if $(S, \cdot, 0)$ is a KU-algebra.

(b) The subset $S$ is said to be an ideal of $A$ if it satisfies the following conditions:

(J1) $0 \in S$, and

(J2) $(\forall x, y, z \in A)((x \cdot(y \cdot z) \in S \wedge y \in S) \Longrightarrow x \cdot z \in S)$.

As shown in [18], this kind of algebra satisfies one specific equality.

Lemma 2 ([18]). In a KU-algebra $A$, the following holds:

$(\mathrm{KU}-5)(\forall x, y, z \in A)(z \cdot(y \cdot x)=y \cdot(z \cdot x))$.

In the light of the previous equality, condition (J2) is transformed into condition:

(J3) $(\forall x, y \in A)((x \cdot y \in S \wedge x \in S) \Longrightarrow y \in S)$.

Indeed, if we put $x=0, y=x$ and $z=y$ in (J2), we immediately obtain (J3) by (KU-2). Conversely, let (J3) be a valid formula and let $x, y, z \in A$ be arbitrary elements such that $x \cdot(y \cdot z) \in J$ and $y \in J$. Then $y \cdot(x \cdot z) \in J$ by (KU-5). Thus $x \cdot z \in J$ by (J3).

From (J3) it immediately follows:

Lemma 3. Let $S$ be an ideal in a KU-algebra $A$. Then

(J4) $(\forall x, y \in A)((x \leqslant y \wedge y \in S) \Longrightarrow x \in S)$.

We can introduce the concept of filters in KU-algebra if formula (J3) serves as a motivation. 
Definition 3. The subset $F$ is said to be a filter of $A$ if it satisfies the following conditions:

(F1) $0 \in F$, and

(F3) $(\forall x, y \in A)((x \cdot y \in F \wedge y \in F) \Longrightarrow x \in F)$.

A filter in KU-algebra, designed in this way, has the following property:

Lemma 4. Let $F$ be a filter in a KU-algebra $A$. Then

(F4) $(\forall x, y \in A)((x \leqslant y \wedge x \in F) \Longrightarrow y \in F)$.

\section{Concept of pseudo-KU algebra}

Definition 4. ([23]) An algebra $\mathfrak{A}=((A, \leqslant), \cdot, *, 0)$ of type $(2,2,0)$ is called a pseudo-KU algebra if it satisfies the following axioms:

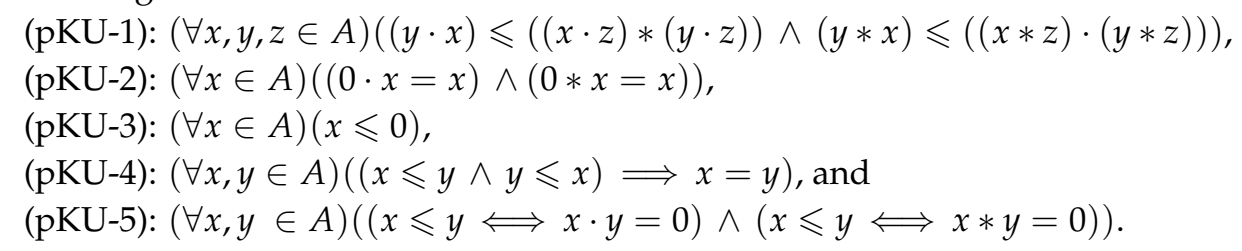

Remark 1. We emphasize that in pseudo-KU algebra the relation of the order is determined inversely with respect to the definition of the order in the KU-algebra.

Lemma 5. If $\mathfrak{A}$ is a pseudo-KU algebra, then

$(\mathrm{pKU}-6)(\forall x \in A)((x \cdot x=0) \wedge(x * x=0))$.

Proof. If we put $x=0, y=0$, and $z=x$ in the formula (pKU-1), we get

$$
(0 \cdot 0) *((0 \cdot x) \star(0 \cdot x))=0 \wedge(0 * 0) \cdot((0 * x) \cdot(0 * x))=0 .
$$

From where we get

$$
x \cdot x=0 \wedge x * x=0
$$

with respect to (pKU-2).

Proposition 1. If $\mathfrak{A}$ is a pseudo-KU algebra, then

(11) $(\forall x, y, z \in A)(x \leqslant y \Longrightarrow((y \cdot z \leqslant x \cdot z) \wedge(y * z \leqslant x * z))$ and

(12) $(\forall x, y, z \in A)(x \leqslant y \Longrightarrow((z \cdot x \leqslant z \cdot y) \wedge(z * x \leqslant z * y))$.

Proof. Let $x, y, z \in A$ such that $x \leqslant y$. Then $x \cdot y=0=x * y$. If we put $x=y$ and $y=x$ in (pKU-1), we get

$$
0=(x \cdot y) *((y \cdot z) *(x \cdot z))=0 *((y \cdot z) *(x \cdot z))=(y \cdot z) *(x \cdot z) .
$$

So, we have $y \cdot z \leqslant x \cdot z$. Similarly, we have

$$
0=(x * y) \cdot((y * z) \cdot(x * z))=0 \cdot((y * z) \cdot(x * z))=(y * z) \cdot(x * z)
$$

and $y * z \leqslant z * x$.

On the other hand, if we put $z=y$ and $y=z$ in (pKU-1), we have

$$
0=(z \cdot x) *((x \cdot y) *(z \cdot y)))=(z \cdot x) *((0 *(z \cdot y))=(z \cdot x) *(z \cdot y) .
$$

This means $z \cdot x \leqslant z \cdot y$. It can be similarly proved that it is $z * x \leqslant z * y$.

In 2011, Mostafa, Naby and Yousef proved Lemma 2.2 in [18]. In the following Proposition, we show that analogous equality is also valid in pseudo-KU algebras.

Proposition 2. In pseudo-KU algebra $\mathfrak{A}$, then

$(\mathrm{pKU})(\forall x, y, z \in A)(x *(y \cdot z)=y *(x \cdot z) \wedge x \cdot(y * z)=y \cdot(x * z))$ 
is valid formula.

Proof. If we put $y=0$ in (pKU-1), we have

$$
0 \cdot x \leqslant(x \cdot z) *(0 \cdot z)
$$

Then, we have $x \leqslant(x \cdot z) * z$. From here it follows

$$
((x \cdot z) * z) \cdot(y * z) \leqslant x \cdot(y * z)
$$

by (11). On the other hand, if we put $x=z \cdot z$ in (pKU-1), we get

$$
y *(x \cdot z) \leqslant((x \cdot z) * z) \cdot(y * z) \leqslant x \cdot(y * z) .
$$

Since the variables $x, y, z \in A$ are free variables, if we put $x=y$ and $y=x$, we get an inverse inequality. From here it follows (pKU) by (pKU-4).

The other equality can be proved in an analogous way.

\section{Correlation of pseudo-KU algebras with other types of pseudo algebras}

The notion of pseudo-UP algebra as a generalization of the concept of UP-algebras was introduced and analyzed in [23].

Definition 5. ([23]) A pseudo-UP algebra is a structure $\mathfrak{A}=((A, \leqslant), \cdot, *, 0)$, where ${ }^{\prime} \leqslant{ }^{\prime}$ is a binary relation on a set $A,{ }^{\prime} \cdot{ }^{\prime}$ and ${ }^{\prime} *$ ' are internal binary operations on $A$ and ${ }^{\prime} 0 '$ is an element of $A$, verifying the following axioms:

$$
\begin{aligned}
& \text { (pUP-1) }(\forall x, y z \in A)(y \cdot z \leqslant(x \cdot y) *(x \cdot z) \wedge y * z \leqslant(x * y) \cdot(x * z)) \\
& \text { (pUP-4) }(\forall x, y \in A)((x \leqslant y \wedge y \leqslant x) \Longrightarrow x=y) ; \\
& (\text { pUP-5) }(\forall x, y \in A)((y \cdot 0) * x=x \wedge(y * 0) \cdot x=x) \text { and } \\
& (\text { pUP-6) }(\forall x, y \in A)((x \leqslant y \Longleftrightarrow x \cdot y=0) \wedge(x \leqslant y \Longleftrightarrow x * y=0)) .
\end{aligned}
$$

The following theorem is an important result of pseudo-KU algebras for study in the connections between pseudo-UP algebras and pseudo-KU algebras.

Theorem 1. Any pseudo-KU algebra is a pseudo-UP algebra.

Proof. It only needs to show (pUP-1). By Proposition 2, we have that any pseudo-KU algebra satisfies (pUP-1).

Pseudo-BE algebra is defined by the follows:

Definition 6. ([3]) An algebra $A=(A, \cdot *, 1)$ of type $(2,2,0)$ is called a pseudo BE-algebra if satisfies in the following axioms:

$$
\begin{aligned}
& \text { (pBE-1) }(\forall x \in A)(x \cdot x=1 \wedge x * x=1) ; \\
& \text { (pBE-2) }(\forall x \in A)(x \cdot 1=1 \wedge x * 1=1) ; \\
& \text { (pBE-3) }(\forall x \in A)(1 \cdot x=x \wedge 1 * x=x) ; \\
& \text { (pBE-4) }(\forall x, y, z \in A)(x \cdot(y * z)=y *(x \cdot z)) ; \text { and } \\
& \text { (pBE-5) }(\forall x, y \in A)(x \cdot y=1 \Longleftrightarrow x * y=1) .
\end{aligned}
$$

If we replace 1 with 0 in (BE-1), (BE-2), (BE-3) and (BE-5) and prove that the formula (pBE-4) is a valid formula in a pseudo-KU algebra $A$, we have proved that every pseudo-KU algebra $A$ is a pseudo-BE algebra.

Theorem 2. Any pseudo-KU algebra is a pseudo-BE algebra.

Proof. It is sufficient to prove that the formula (pBE-4) is a valid formula in any pseudo-KU algebra. If we put $y=0$ in the left-hand side of the formula (pKU-1), we get $0 \cdot x \leqslant((x \cdot z) *(0 \cdot z)$. It means $x \leqslant(x \cdot z) * z$. From here follows

$$
((x \cdot z) * z) \cdot(y * z) \leqslant x \cdot(y * z),
$$


by the left part of formula (11). On the other hand, if we put $x=x \cdot z$ in the right-hand side of the formula (pKU-1), we get

$$
y *(x \cdot z) \leqslant((x \cdot z) * z) \cdot(y * z)
$$

Which together with the previous inequality gives

$$
y *(x \cdot z) \leqslant x \cdot(y * z)
$$

From this inequality by substituting the variables $x$ and $y$, we obtain the necessary reverse inequality

$$
x \cdot(y * z) \leqslant y *(x \cdot z)
$$

From these two inequalities follows the validity of the formula (pBE-4) in any pseudo-KU algebra by the axiom (pKU-4).

Since the formula previously proven is important below, we point it out in particular.

Proposition 3. In any pseudo-KU algebra $\mathfrak{A}$,

$($ pKU-7) $(\forall x, y, z \in A)(x \cdot(y * z)=y *(x \cdot z))$

is a valid formula.

\section{Some substructures in pseudo-KU algebras}

\subsection{Concept of pseudo-subalgebras}

Definition 7. A nonempty subset $S$ of a pseudo-KU algebra $A$ is a pseudo-subalgebra in $\mathfrak{A}$ if

$$
(\forall x, y \in A)((x \in S \wedge y \in S) \Longrightarrow(x \cdot y \in S \wedge x * y \in S)) .
$$

holds.

Putting $y=x$ in the previous definition, it immediately follows:

Lemma 6. If $S$ is a pseudo-subalgebra of a pseudo-KU algebra $\mathfrak{A}$, then $0 \in S$.

Proof. Let $S$ be a pseudo-subalgebra of a pseudo-KU algebra $\mathfrak{A}$. It means that $S$ is a nonempty subset of $A$. Then there exists an element $y \in S$. Thus $0=y \cdot y=y * y \in S$ by Definition 7 .

It is clear that subsets $\{0\}$ and $A$ are pseudo-subalgebras of a pseudo-KU algebras $\mathfrak{A}$. So, the family $\mathfrak{S}(A)$ of all pseudo-subalgebras of a pseudo-KU algebra $\mathfrak{A}$ is not empty. Without major difficulties, the following theorem can be proved.

Theorem 3. The family $\mathfrak{S}(A)$ of all pseudo-subalgebras of a pseudo-KU algebra $\mathfrak{A}$ forms a complete lattice.

\subsection{Concept of pseudo-ideals}

Definition 8. The subset $J$ is said to be a pseudo-ideal of a pseudo-KU algebra $\mathfrak{A}$ if it satisfies the following conditions:

(pJ1) $0 \in J$,

(pJ3a) $(\forall x, y \in A)((x \cdot y \in J \wedge x \in J) \Longrightarrow y \in J)$ and

(pJ3b) $(\forall x, y \in A)((x * y \in J \wedge x \in J) \Longrightarrow y \in J)$.

Proposition 4. Let $J$ be a nonempty subset of a pseudo-KU algebra $\mathfrak{A}$. Then the condition ( $p J 3 a)$ is equivalent to the condition:

$$
(\mathrm{pJ} 4 \mathrm{a})(\forall x, y, z \in A)((x *(y \cdot z) \in J \wedge y \in J) \Longrightarrow x * z \in J) .
$$

Proof. Putting $x=y$ and $y=x * z$ in the condition (pJ3a), it immediately follows

$$
(\forall x, y, z \in A)((y \cdot(x * z) \in J \wedge y \in J) \Longrightarrow x * z \in J) .
$$


Thus

$$
(\forall x, y, z \in A)((y *(x \cdot z) \in J \wedge y \in J) \Longrightarrow x * z \in J)
$$

by (pKU-7).

Conversely, let (pJ4a) it be. Let us choose $x=0, y=x$ and $z=y$ in (pJ4a). We get $(0 *(x \cdot y) \in J \wedge x \in$ $J) \Longrightarrow 0 * y \in J$. Thus (pJ3a) by (pKU-2).

Corollary 4. Let $J$ be a pseudo-ideal in a pseudo-KU-algebra $\mathfrak{A}$. Then

(13) $(\forall x, y \in A)(y \in J \Longrightarrow x * y \in J)$.

Proof. Putting $z=y$ in (pJ4a), with respect to (pKU-6), (pKU-3) and (pJ1), we obtain (13).

Proposition 5. Let J be a nonempty subset of a pseudo-KU algebra $\mathfrak{A}$. Then the condition ( $p J 3 b)$ is equivalent to the condition

(pJ4b) $(\forall x, y, z \in A)((x \cdot(y * z) \in J \wedge y \in J) \Longrightarrow x \cdot z)$.

Proof. If we put $x=y$ and $y=x \cdot z$ in (pJ3b), we get

$$
(y *(x \cdot z) \in J \wedge y \in J) \Longrightarrow x \cdot z \in J .
$$

Hence

$$
(x \cdot(x * z) \in J \wedge y \in J) \Longrightarrow x \cdot z \in J
$$

by (pKU-7).

Conversely, if we put $x=0, y=x$, and $z=y$ in (pJ4b), we get

$$
(0 \cdot(x * y) \in J \wedge x \in J) \Longrightarrow 0 \cdot y \in J .
$$

Thus (pJ3b) with respect to (pKU-2).

Corollary 5. Let $\mathrm{J}$ be a pseudo-ideal in a pseudo-KU-algebra $\mathfrak{A}$. Then

(14) $(\forall x, y \in A)(y \in J \Longrightarrow x \cdot y \in J)$.

Proof. Putting $z=y$ in (pJ4b), with respect to (pKU-6), (pKU-3) and (pJ1), we obtain (14).

The following important statement describes the connection between conditions (pJ3a) and (pJ3b).

Proposition 6. Let J be a pseudo-ideal of a pseudo-KU algebra $\mathfrak{A}$. Then

$$
(p J 3 a) \Longleftrightarrow(p J 3 b) .
$$

Proof. $(p J 3 a) \Longleftrightarrow(p J 3 b)$. Suppose (pJ3a) holds and let $x * y \in J$ and $x \in J$. How obvious it is that the following

$$
x *((x \cdot y) * y)=0 \Longleftrightarrow x \cdot((x \cdot y) \star x)=0 \Longleftrightarrow(x \cdot y) *(x \cdot y)=0
$$

is valid, we have

$$
(x \in J \wedge x \cdot((x * u) \cdot y)=0 \in J) \Longrightarrow(x * y) \cdot y \in J .
$$

Now

$$
(x * y \in J \wedge(x * y) \cdot y \in J) \Longrightarrow y \in J .
$$

We have proved that (pJ3b) is a valid implication.

$(p J 3 b) \Longrightarrow(p J 3 a)$. Let (pJ3b) be a valid formula and let $x, y \in A$ be such that $x \in J$ and $x \cdot y \in J$. As above, from

$$
x *((x \cdot y) * y)=0 \Longleftrightarrow x \cdot((x \cdot y) * y)=0 \Longleftrightarrow(x \cdot y) *(x \cdot y)=0
$$

it follows

$$
(x \in J \wedge x *((x \cdot y) * y)=0 \in J) \Longrightarrow(x \cdot y) * y \in J .
$$

Now, $x \cdot y \in J$ and $(x \cdot y) * y$ it follows $y \in J$. This proves the validity of the formula (pJ3a). 
Proposition 7. Any pseudo-ideal in a pseudo-KU-algebra $\mathfrak{A}$ is a pseudo-subalgebra in $\mathfrak{A}$.

Proof. The proof of this proposition follows from (13) and (14).

Theorem 6. The family $\mathfrak{J}(A)$ of all pseudo-ideals in a pseudo-KU algebra $\mathfrak{A}$ forms a complete lattice and $\mathfrak{J}(A) \subseteq \mathfrak{S}(A)$ holds.

Proof. Let $\left\{J_{i}\right\}_{i \in I}$ be a family of pseudo-ideals in a pseudo-KU algebra $\mathfrak{A}$. Clearly $0 \in \bigcap_{i \in I} J_{i}$ is valid. Let $x, y \in A$ be elements such that $x \cdot y \in \bigcap_{i \in I} J_{i}, x * y \in \bigcap_{i \in I} J_{i}$ and $x \in \bigcap_{i \in I} J_{i}$. Then $x \cdot y \in J_{i}, x * y \in J_{i}$ and $x \in F_{i}$ for any $i \in I$. Thus $y \in J_{i}$ because $J_{i}$ is a pseudo-ideal in $\mathfrak{A}$ and $x \in \bigcap_{i \in I} J_{i}$. So, $\bigcap_{i \in I} J_{i}$ is a pseudo-ideal in $\mathfrak{A}$.

If $\mathfrak{X}$ is the family of all pseudo-ideals of $\mathfrak{A}$ that contain the union $\bigcup_{i \in I} J_{i}$, then $\cap \mathfrak{X}$ is also a pseudo-ideal in $\mathfrak{A}$ that contains $\bigcup_{i \in I} J_{i}$ by previous evidence.

If we put $\Pi_{i \in I} J_{i}=\bigcap_{i \in I} J_{i}$ and $\sqcup_{i \in I} J_{i}=\cap \mathfrak{X}$, then $(\mathfrak{J}(A), \sqcap, \sqcup)$ is a complete lattice.

To round out this subsection we need the following lemma.

Lemma 7. Let $J$ be a pseudo-ideal in a pseudo-KU algebra $\mathfrak{A}$. Then

(15) $(\forall x, y \in A)((x \leqslant y \wedge x \in J) \Longrightarrow y \in J)$.

Proof. The proof of this proposition follows from (pJ3a) (or (pJ3b)) with respect to (pKU-6) and (pJ1).

Theorem 7. Let $J$ be a subset of a pseudo-KU algebra $\mathfrak{A}$ such that $0 \in J$. Then, $J$ is a pseudo-ideal in $\mathfrak{A}$ if and only if the following holds

(pJ5) $(\forall x, y, z \in A)((x \in A \wedge y \in A \wedge x \leqslant y \cdot z) \Longrightarrow z \in J)$.

Proof. Let $J$ be a pseudo-ideal in $\mathfrak{A}$ and let $x, y, z \in A$ such that $x \in J, y \in J$ and $x \leqslant y \cdot z$. Then $x \cdot(y \cdot z)=0 \in J$. Thus $y \cdot z \in J$ by (pJ3a) and again, from here and $y \in J$ it follows $z \in J$. So, we have shown that (pJ5) is a valid formula.

Opposite, suppose that (pJ5) is a valid in $\mathfrak{A}$. Let us show that $J$ is a pseudo-ideal and $\mathfrak{A}$. Let $x, y \in A$ be such that $x \in J$ and $x \cdot y \in J$. Then $x * y \in J$ by Proposition 6. On the other hand, from $x \cdot((x * y) \cdot y)=0$, i.e. from $x \leqslant(x * y) \cdot y$ it follows $y \in J$ by hypothesis. So, the set $J$ is a pseudo-ideal in $\mathfrak{A}$.

For a relation on the set $A$ we say that it is a quasi-order relation on $A$ if it is reflexive and transitive. It is easy to prove that if $\sigma$ is a quasi-order relation on $A$, then the relation $\sigma \cap \sigma^{-1}$ is an equivalence on $A$.

Theorem 8. Let J be a pseudo-ideal in a pseudo-KU algebra $\mathfrak{A}$. Then the relation' $\preccurlyeq{ }^{\prime}$, defined by

$$
(\forall x, y \in A)(x \preccurlyeq y \Longleftrightarrow x \cdot y \in J),
$$

is a quasi-order in the set A left compatible and right reverse compatible with the internal operations in $\mathfrak{A}$.

Proof. Since $x \cdot x=0 \in J$ is valid in $\mathfrak{A}$ for any $x \in A$, it is clear that ${ }^{\prime} \preccurlyeq{ }^{\prime}$ is a reflexive relation in the set $A$.

Let $x, y, z \in A$ be arbitrary elements such that $x \preccurlyeq y$ and $y \preccurlyeq z$. This means $x \cdot y \in J$ and $y \cdot z \in J$. From inequality (pKU-1) in the form $x \cdot y \leqslant(y \cdot z) *(x \cdot z)$ and $x \cdot y \in J$ it follows $(y \cdot z) *(x \cdot z) \in J$ according to (15). From here and from $y \cdot z \in J$ it follows $x \cdot z \in J$ according to (pJ3a). Hence, the relation ' $\preccurlyeq$ ' is transitive. So, this relation is a quasi-order in $A$.

Let $x, y, z \in A$ be such $x \preccurlyeq y$. Then $x \cdot y \in J$ and $x * y \in J$.

(i) If we put $x=y$ and $y=x$ in the left part of the formula (pKU-1), we get $x \cdot y \leqslant(y \cdot z) *(x \cdot z)$. Now, from here and $x \cdot y \in J$ it follows $(y \cdot z) *(x \cdot z) \in J$ by (15). Thus $(y \cdot z) \cdot(x \cdot z) \in J$ by Proposition 6. Finally, we have $y \cdot z \preccurlyeq x \cdot z$. So, the relation ${ }^{\prime} \preccurlyeq{ }^{\prime}$ is reverse right compatible with the internal operation ${ }^{\prime} \cdot{ }^{\prime}$ in $\mathfrak{A}$.

(ii) If we put $x=y$ and $y=x$ in the right part of the formula (pKU-1), we get $x * y \leqslant(y * z) \cdot(x * z)$. Then $(y * z) \cdot(x * z) \in J$ by (15). Thus $y * z \preccurlyeq x * z$. Therefore, the relation ${ }^{\prime} \preccurlyeq{ }^{\prime}$ is reverse right compatible with the internal operation' $*^{\prime}$ in $\mathfrak{A}$.

(iii) Let us put $y=z$ and $z=y$ in the left part of the formula (pKU-1). We get $(z \cdot x) *((x \cdot y) *(z \cdot y))=$ $0 \in J$. From here and from $x \cdot y \in J$ it follows $(z \cdot x) *(z \cdot y) \in J$ by (pJ4a). Thus $z \cdot x \preccurlyeq z \cdot y$. So, the relation $' \preccurlyeq '$ is left compatible with the operation '. '. 
(iv) Let us put $y=z$ and $z=y$ in the right part of the formula (pKU-1). We get $(z * x) \cdot((x * y) \cdot(z * y))=$ $0 \in J$. From here and from $x * y \in J$ it follows $(z * x) \cdot(z * y) \in J$ by (pJ4b). Thus $z * x \preccurlyeq z * y$. So, the relation $' \preccurlyeq{ }^{\prime}$ is left compatible with the operation ${ }^{\prime} * '$.

\subsection{Concept of pseudo-filters}

Definition 9. A non-empty subset $F$ of a pseudo-KU algebra $\mathfrak{A}$ is called a pseudo-filter of $A$ if it satisfies in the following axioms:

(pF1) $0 \in F$;

(pF3) $(\forall x, y \in A)((x \cdot y \in F \wedge x * y \in F \wedge y \in F) \Longrightarrow x \in F)$.

$\{0\}$ and $A$ are pseudo-filters of $\mathfrak{A}$. So, the family $\mathfrak{F}(A)$ of all pseudo-filters in a pseudo-KU algebra $\mathfrak{A}$ is not empty.

It is obviously the following is valid

Lemma 8. Let $F$ be a pseudo-filter in a pseudo-KU algebra $\mathfrak{A}$. Then

(16) $(\forall x, y \in A)((x \leqslant y \wedge y \in F) \Longrightarrow x \in F)$.

Theorem 9. The family $\mathfrak{F}(A)$ of all pseudo-ideals in a pseudo-KU algebra $\mathfrak{A}$ forms a complete lattice.

Proof. Let $\left\{F_{i}\right\}_{i \in I}$ be a family of pseudo-filters in a pseudo-KU algebra $\mathfrak{A}$. Clearly $0 \in \bigcap_{i \in I} F_{i}$ is valid. Let $x, y \in A$ be elements such that $x \cdot y \in \bigcap_{i \in I} F_{i}, x * y \in \bigcap_{i \in I} F_{i}$ and $y \in \bigcap_{i \in I} F_{i}$. Then $x \cdot y \in F_{i}, x * y \in F_{i}$ and $y \in F_{i}$ for any $i \in I$. Thus $x \in F_{i}$ because $F_{i}$ is a pseudo-filter in $\mathfrak{A}$ and $x \in \bigcap_{i \in I} F_{i}$. So, $\bigcap_{i \in I} F_{i}$ is a pseudo-filter in $\mathfrak{A}$.

If $\mathfrak{X}$ is the family of all pseudo-filters of $\mathfrak{A}$ that contain the union $\bigcup_{i \in I} F_{i}$, then $\cap \mathfrak{X}$ is also a pseudo-filter in $\mathfrak{A}$ that contains $\bigcup_{i \in I} F_{i}$ by previous evidence.

If we put $\prod_{i \in I} F_{i}=\bigcap_{i \in I} F_{i}$ and $\sqcup_{i \in I} F_{i}=\cap \mathfrak{X}$, then $(\mathfrak{F}(A), \sqcap, \sqcup)$ is a complete lattice.

\section{Concept of pseudo-homomorphisms}

Definition 10. $\left((A, \leqslant A), \cdot_{A}, *_{A}, 0_{A}\right)$ and $\left(\left(B, \leqslant_{B}\right), \cdot_{B}, *_{B}, 0_{B}\right)$ be pseudo-KU algebras. A mapping $f: A \longrightarrow B$ of pseudo-KU algebras is called a pseudo-homomorphism if

$$
(\forall x, y \in A)\left(f(x \cdot A y)=_{B} f(x) \cdot_{B} f(y) \wedge f\left(x *_{A} y\right)=_{B} f(x) *_{B} f(y)\right) .
$$

Remark 2. Note that if $f: A \longrightarrow B$ is a pseudo homomorphism, then $f\left(0_{A}\right)=0_{B}$. Indeed, if we chose $y=x$, from the previous formula we immediately get $f\left(0_{A}\right)={ }_{B} 0_{B}$ with respect (pKU-6).

From here it immediately follows:

Lemma 9. Any pseudo-homomorphism between pseudo-KU algebras is isotone mapping.

Proof. Let $f: A \longrightarrow B$ be a pseudo-homomorphism between pseudo-KU algebras and let $x, y \in A$ be such $x \leqslant{ }_{A} y$. Then $x \cdot{ }_{A} y={ }_{A} 0_{A}$. Thus $0_{B}=_{B} f(x \cdot A y)=_{B} f(x) \cdot{ }_{B} f(y)$. This means $f(x) \leqslant_{B} f(y)$.

Lemma 10. Let $f: A \longrightarrow B$ be a pseudo-homomorphism between pseudo-KU algebras. Then the set $\operatorname{Ker}(f)={ }_{A}\{x \in$ $\left.A: f(x)={ }_{B} 0_{B}\right\}$ is a pseudo-ideal in $\mathfrak{A}$.

Proof. It is obvious $0_{A} \in \operatorname{Ker}(f)$.

Let $x, y \in A$ be such $x \cdot{ }_{A} y \in \operatorname{Ket}(f)$ and $x \in \operatorname{Ker}(f)$. Then $f(x)={ }_{B} 0_{B}$ and $0=_{B} f(x \cdot A y)={ }_{B} f(x) \cdot{ }_{B}$ $f(y)={ }_{B} 0_{B} \cdot B f(y)={ }_{B} f(y)$. Thus $y \in \operatorname{Ker}(f)$.

The implication of $x *_{A} y \in \operatorname{Ker}(f) \wedge x \in \operatorname{Ker}(f) \Longrightarrow y \in \operatorname{Ker}(f)$ can be proved by analogy with the previous proof.

The following statement is easy to prove:

Lemma 11. If $f: A \longrightarrow B$ is a pseudo-homomorphism between pseudo-KU algebras, then $f(A)$ is a pseudo-subalgebra in $B$. 
Proposition 8. Let $f: A \longrightarrow B$ be a pseudo homomorphism between pseudo-KU algebras $\mathfrak{A}$ and $\mathfrak{B}$.

(i) If $K$ is a pseudo-ideal in $\mathfrak{B}$, then $f^{-}(K)$ is a pseudo-ideal in $\mathfrak{A}$.

(ii) If $G$ is a pseudo-filter in $\mathfrak{B}$, then $f^{-1}(G)$ is a pseudo-filter in $\mathfrak{A}$.

Proof. (i) Assume that $K$ is a pseudo-fulter of $\mathfrak{B}$. Obviously $0_{A} \in f^{-1}(K)$. Let $x, y \in A$ be such $x \cdot y \in f^{-1}(K)$ and $x \in f^{-1}(K)$. Then $f(x) \cdot{ }_{B} f(y)=_{B} f(x \cdot A y) \in K$ and $f(x) \in K$. It follows that $f(y) \in K$ by (pJ3a) since $K$ is a pseudo-ideal in $\mathfrak{B}$. Therefore, $y \in f^{-1}(K)$. Thus, the set $f^{-1}(K)$ satisfies the implication (pJ3a). That the set $f^{-1}(K)$ satisfies the implication (pJ3b) can be proved in an analogous way. Therefore, the set $f^{-1}(K)$ is a pseudo-ideal in $\mathfrak{A}$.

(ii) It is obvious $0_{A} \in f^{-1}(G)$ again. Let $x, y \in A$ be elements such that $x \cdot A y \in f^{-1}(G), x *_{A} y \in f^{-1}(G)$ and $y \in f^{-1}(G)$. Then $f(x) \cdot \cdot_{B} f(y)=_{B} f(x \cdot A y) \in G, f(x) *_{B} f(y)=_{B} f\left(x *_{A} y\right) \in G$ and $f(y) \in G$. Thus $f(x) \in G$ because $G$ is a pseudo-filter in $\mathfrak{B}$. This means $x \in f^{-1}(G)$. So, the $\operatorname{set}^{-1}(G)$ is a pseudo-filter in $\mathfrak{A}$.

In the following definition, we will introduce the concept of congruence on pseudo-KU algebras. Since we have two unitary operations on this algebra, it is possible to determine three different types of congruences.

Definition 11. Let $\mathfrak{A}=((A, \leqslant), \cdot, *, 0)$ be a pseudo-KU algebra.

For the equivalence relation $q$ on the set $A$ we say that it is a congruence of type ' . ' on $\mathfrak{A}$ if it compatible with the operations' ${ }^{\prime}{ }^{\prime}$ in $\mathfrak{A}$ in the following sense

(17) $(\forall x, y, z \in A)((x, y) \in q \Longrightarrow((x \cdot z, y \cdot z) \in q \wedge(z \cdot x, z \cdot y) \in q)))$.

For the equivalence relation $q$ on the set $A$ we say that it is a congruence of type' $*^{\prime}$ on $\mathfrak{A}$ if it compatible with the operations ' * ' in $\mathfrak{A}$ in the following sense

(18) $(\forall x, y, z \in A)((x, y) \in q \Longrightarrow((x * z, y * z) \in q \wedge(z * x, z * y) \in q)))$.

For the equivalence relation $q$ on the set $A$ we say that it is a congruence of common type on $\mathfrak{A}$ if it is compatible with both operations in $\mathfrak{A}$.

Lemma 12. Let $q$ be a relation on a pseudo-KU algebra $\mathfrak{A}$. Then:

(i) The condition (17) is equivalent to the condition

(17a) $(\forall x, y, u, v \in A)(((x, y) \in q \wedge(u, v) \in q) \Longrightarrow(x \cdot u, y \cdot v) \in q)$.

(ii) The condition (18) is equivalent to the condition

(18a) $(\forall x, y, u, v \in A)(((x, y) \in q \wedge(u, v) \in q) \Longrightarrow(x * u, y * v) \in q)$.

Proof. $(17 a) \Longrightarrow(17)$. If we choose $v=z$ in (17a), we get the implication $(x, y) \in q \Longrightarrow(x \cdot z, y \cdot z) \in q$. On the other hand, if we put $x=y=z, u=x$ and $v=y$ in (17a), we get the implication $(x, y) \in q \Longrightarrow(z \cdot x, z \cdot t)$.

$(17) \Longrightarrow(17 a)$. Suppose (17) and let $x, y, u, v \in A$ such that $(x, y) \in q$ and $(u, v) \in q$. Thus $(x \cdot u, x \cdot v) \in q$ and $(x \cdot v, y \cdot v) \in q$ by $(16)$. Hence $(x \cdot u, y \cdot v) \in q$ by transitivity of $q$.

Equivalence $(18) \Longleftrightarrow(18 a)$ can be proved analogous to the previous proof.

Let $f: A \longrightarrow B$ be a pseudo homomorphism between pseudo-KU algebras. By direct check without difficulty, it can be proved that the relation $q_{f}$, defined by

$$
(\forall x, y \in A)\left((z, y) \in q_{f} \Longleftrightarrow f(x)={ }_{B} f(y)\right),
$$

is a congruence (all three types) on $\mathfrak{A}$.

Theorem 10. The relation $q_{f}$ is a congruence of type' . ' (type ${ }^{\prime} *$ ', common type) on the pseudo-KU algebra $\mathfrak{A}$.

Proof. We will only demonstrate the proof that $q_{f}$ is a congruence of type ${ }^{\prime} . '$ on $\mathfrak{A}$ because the evidence that $q_{f}$ is a congruence of type' $*^{\prime}$ can obtain by analogy with the previous one, and the proof of common type is obtained by combining this two evidences.

Clearly, $q_{f}$ is an equivalence relation on the set $A$. It remains to verify that (16) is a valid formula in $\mathfrak{A}$. Let $x, y, u, v \in A$ be such that $(x, y) \in q_{f}$ and $(u, v) \in q_{f}$. Then $f(x)={ }_{B} f(y)$ and $f(u)=_{B} f(v)$. Thus

$$
f\left(x \cdot{ }_{A} u\right)={ }_{B} f(x) \cdot{ }_{B} f(u)={ }_{B} f(y) \cdot{ }_{B} f(v)={ }_{B} f(y \cdot A u) .
$$


Hence, $\left(x \cdot A u, y \cdot{ }_{A} v\right) \in q_{f}$. We proved that (17a) is a valid formula. So $q_{f}$ is a congruence of type ${ }^{\prime} \cdot{ }^{\prime}$ on $\mathfrak{A}$.

Theorem 11. Let $J$ be a pseudo-ideal in a pseudo-KU algebra $\mathfrak{A}$. Then the relation $q_{J}$, defined by $q_{J}=\preccurlyeq \cap \preccurlyeq^{-1}$, is a congruence of common type in $\mathfrak{A}$.

Proof. The relation $q$ is an equivalence relation on the set $A$. It is sufficient to prove that $q$ is compatible with operations in $\mathfrak{A}$. Since the relation $\preccurlyeq$ is left compatible and right reverse compatible with the internal operations in $\mathfrak{A}$, by Theorem 8 , it is clear that the relation $q_{J}$ is a congruence on $\mathfrak{A}$.

For a congruence $q$ on a pseudo-KU algebra $\mathfrak{A}$ we denote $q x=\{y \in A:(x, y) \in q\}=[x]$. Let's define '• ' and ' $\star$ ' in $A / q$ on this way

$$
(\forall x, y \in A)([x] \bullet[y]=[x \cdot y]) \text { and }(\forall x, y \in A)([x] \star[y]=[x * y]) .
$$

Without much difficulty it can be verified that the functions ' $\bullet$ ' and ' $\star$ ', defined in this way, are well-defined internal binary operations in $A / q$. Also, one can check that the set $A / q$ with the operations ' ' and ' $\star$ ', determined as above, satisfies all the axioms of Definition 4 except the axiom (pKU-4). However, if we take the relation $q_{J}$, defined by an pseudo-ideal $J$ of a pseudo-KU algebra $\mathfrak{A}$, then we have

Theorem 12. Let J be a pseudo-ideal in a pseudo-KU algebra $\mathfrak{A}$. Then the structure $((A / q, \leqq), \bullet, \star,[0])$, where' $\leqq{ }^{\prime}$ is defined by

$$
(\forall x, y \in A)([x] \leqq[y], \Longleftrightarrow x \preccurlyeq y),
$$

is a pseudo-KU algebra, too.

Proof. According to the commentary preceding this theorem, to prove this theorem it suffices to show that the structure $((A / q, \leqq), \bullet, \star,[0])$ satisfies the axiom (pKU-4).

Let $x, y \in A$ be such $[x] \leqq[y]$ and $[y] \leqq[x]$. Then $x \preccurlyeq y$ and $y \preccurlyeq x$ by definition. Thus $(x, y) \in q_{J}$ and $[x]=[y]$.

Let $f: A \longrightarrow B$ be pseudo-homomorphism between pseudo-KU algebras $\left((A, \leqslant A), \cdot_{A}, *_{A}, 0_{A}\right)$ and $\left((B, \leqslant B), \cdot_{B}, *_{B}, 0_{B}\right)$. Then the set $f(A)$ is a pseudo-subalgebra of $\mathfrak{B}$ by and the set $J=\operatorname{Ker}(f)$ is a pseudo-ideal in $\mathfrak{A}$ by Lemma 10 and the relation $q_{f}$ is a congruence on $\mathfrak{A}$ by Theorem 10. If $(x, y) \in q_{f}$ holds soe some $x, y \in A$, we have $f(x)={ }_{B} f(y)$. Thus $f(x \cdot A y)=_{B} f(x) \cdot{ }_{B} f(y)={ }_{B} f(x) \cdot{ }_{B} f(x)={ }_{B} 0_{B}$, i.e. $x \cdot A y \in J$. Analogous to the previous one may be shown that $y \cdot{ }_{A} x \in J$ holds. Thus, $(x, y) \in q_{f} \Longrightarrow(x, y) \in q_{J}$ is valid.

We end this section with the following theorem. Since this theorem can be proven by direct verification, we will omit evidence for it.

Theorem 13. Let $f: A \longrightarrow B$ be pseudo-homomorphism between pseudo-KU algebras $\left(\left(A, \leqslant_{A}\right), \cdot_{A}, *_{A}, 0_{A}\right)$ and $\left(\left(B, \leqslant_{B}\right), \cdot_{B}, *_{B}, 0_{B}\right)$. Then there exists the unique epimorphism $\pi: A \longrightarrow A / q_{f}$, defined by $\pi(x)=[x]$ for any $x \in A$, and the unique monomorphism $g: A / q_{f} \longrightarrow B$, defined by $g([x])={ }_{B} f(x)$ for any $x \in A$ such that $f=g \circ \pi$.

Acknowledgments: The author thanks the reviewers for helpful suggestions. The author also thanks the editors of the journal for their assistance in the technical preparation of the manuscript.

Conflicts of Interest: "The author declares no conflict of interest."

\section{References}

[1] Georgescu, G., \& Iorgulescu, A. (2001). Pseudo-BCK algebras: an extension of BCK algebras. In Combinatorics, computability and logic (pp. 97-114). Springer, London.

[2] Dudek, W. A., \& Jun, Y. B. (2008). Pseudo-BCI algebras. East Asian Mathematical Journal, 24(2), 187-190.

[3] Borzooei, R., Saeid, A., Rezaei, A., Radfar, A., \& Ameri, R. (2013). On pseudo BE-algebras. Discussiones Mathematicae-General Algebra and Applications, 33(1), 95-108.

[4] Ciungu, L. C. (2016). Commutative pseudo BE-algebras. Iranian Journal of Fuzzy Systems, 13(1), 131-144.

[5] Dymek, G. (2008). Some properties of pseudo-BCI algebras. Commentationes Mathematicae, 58(1-2), 19-35.

[6] Emanovský, P., \& Kühr, J. (2018). Some properties of pseudo-BCK-and pseudo-BCI-algebras. Fuzzy Sets and Systems, $339,1-16$.

[7] Iorgulescu, A. (2004). On pseudo-BCK algebras and porims. Scientiae Mathematicae Japonicae, 60(3), 501-514. 
[8] Iorgulescu, A. (2006). Classes of pseudo-BCK algebras: Part I. Journal of Multiple-Valued Logic and Soft Computing, 12, 71-130.

[9] Walendziak, A. (2011). On axiom systems of pseudo-BCK algebras. Bulletin of the Malaysian Mathematical Sciences Society, Second Series, 34(2), 287-293.

[10] Xin, X. L., Li, Y. J., \& Fu, Y. L. (2017). States on pseudo-BCI algebras. European Journal of Pure and Applied Mathematics, 10(3), 455-472.

[11] Hájek, P. (1998). Basic fuzzy logic and BL-algebras. Soft computing, 2(3), 124-128.

[12] Di Nola, A., Georgescu, G., \& Iorgulescu, A. (2002). Pseudo-BL algebras: part I. Multiple Valued Logic, 8(5/6), 673-716.

[13] Di Nola, A., Georgescu, G. and Iorgulescu, A. (2002). Pseudo-BL algebras: part II. Multiple-Valued Logic, 8(5-6), 717-750.

[14] Zhang, X. H., \& Li, W. H. (2006). On pseudo-BL algebras and BCC-algebras. Soft Computing, 10, Article No. 941.

[15] Prabpayak, C., \& Leerawat, U. (2009). On ideals and congruences in KU-algebras. Scientia Magna journal, 5(1), 54-57.

[16] Prabpayak, C., \& Leerawat, U. (2009). On isomorphisms of KU-algebras. Scientia Magna journal, 5(3), 25-31.

[17] Kareem, F. F., \& Hasan, E. R. (2018). On KU-semigroups. International Journal of Science and Nature, 9(1), 79-84.

[18] Mostafa, S. M., Abd-Elnaby, M. A., \& Yousef, M. M. (2011). Fuzzy ideals of KU-Algebras. International Mathematical Forum, 6(63), 3139-3149.

[19] Iampan, A. (2017). A new branch of the logical algebra: UP-algebras. Journal of Algebra and Related Topics, 5(1), 35-54.

[20] Rezaei, A., Saeod, A. B., \& Borzooei, R. A. (2014). KU-algebras are equivalent to commutative self-distributive BE-algebras. Bollettino di Matematica Pura e Applicata, 7, 1-8.

[21] Kim, H. S., \& Kim, Y. H. (2007). On BE-algebras. Scientiae Mathematicae Japonicae, 66(1), 113-116.

[22] Rezaei, A., \& Saeid, A. B. (2012). Some results in BE-algebras. Analele Universitatii din Oradea. Fascicola Matematica, 19(1), 33-44.

[23] Romano, D. A. (2020). Pseudo-UP algebras, An introduction. Bulletin of the International Mathematical Virtual Institute, 10(2), 349-355.

[24] Leerawat, U., \& Prabpayak, C. (2015). Pseudo KU-algebras and their applications in topology. Global Journal of Pure and Applied Mathematics, 11(4), 1793-1801.

[25] Ali, U., Ansari, M. A., \& Rehman, M. U. (2019). Pseudo-valuations and pseudo-metric on JU-algebras. Open Journal of Mathematical Sciences, 3(1), 440-446.

(C) 2020 by the authors; licensee PSRP, Lahore, Pakistan. This article is an open access article distributed under the terms and conditions of the Creative Commons Attribution (CC-BY) license (http://creativecommons.org/licenses/by/4.0/). 